\title{
Study of the LOFAR radio self-trigger and single-station acquisition mode
}

\author{
A. Bonardi ${ }^{* 1}$, S. Buitink ${ }^{2}$, A. Corstanje ${ }^{1}$, H. Falcke ${ }^{1,3,4}$, B.M. Hare ${ }^{5}$, J.R. Hörandel ${ }^{1,3}$, \\ P. Mitra ${ }^{2}$, K. Mulrey ${ }^{2}$, A. Nelles ${ }^{1,6}$, J.P. Rachen ${ }^{1}$, L. Rossetto ${ }^{1}$, P. Schellart ${ }^{1,8}$, \\ O. Scholten ${ }^{5,7}$, S. ter Veen ${ }^{1,4}$, S. Thoudam ${ }^{1,9}$, T.N.G. Trinh ${ }^{5}$, T. Winchen ${ }^{2}$ \\ ${ }^{1}$ Department of Astrophysics/IMAPP, Radboud University, P.O. Box 9010, 6500 GL Nijmegen, \\ The Netherlands \\ ${ }^{2}$ Astrophysical Institute, Vrije Universiteit Brussel, Pleinlaan 2, 1050 Brussels, Belgium \\ ${ }^{3}$ NIKHEF, Science Park Amsterdam, 1098 XG Amsterdam, The Netherlands \\ ${ }^{4}$ Netherlands Institute of Radio Astronomy (ASTRON), Postbus 2, 7990 AA Dwingeloo, The \\ Netherlands \\ ${ }^{5}$ KVI-CART, University Groningen, P.O. Box 72, 9700 AB Groningen, The Netherlands \\ ${ }^{6}$ Department of Physics and Astronomy, University of California Irvine, Irvine, CA 92697-4575, \\ USA \\ ${ }^{7}$ Interuniversity Institute for High-Energy, Vrije Universiteit Brussel, Pleinlaan 2, 1050 \\ Brussels, Belgium \\ ${ }^{8}$ Department of Astrophysical Sciences, Princeton University, Princeton, NJ 08544, USA \\ ${ }^{9}$ Department of Physics and Electrical Engineering, Linnéuniversitetet, 35195 Växjö, Sweden \\ E-mail: a.bonardieastro.ru.nl
}

The LOw Frequency ARay (LOFAR) observatory is a multipurpose radio antenna array aimed to detect radio signals in the frequency range $10-240 \mathrm{MHz}$. Radio antennas are clustered into over 50 stations, and are spread along Central and Northern Europe. The LOFAR core, where the density of stations is highest, is instrumented with the LOfar Radboud air shower Array (LORA), covering an area of about $300 \mathrm{~m}$ diameter centered at the LOFAR core position. Since 2011 the LOFAR core has been used for detecting radio-signals associated to cosmic-ray air showers in the energy range $10^{16}-10^{18} \mathrm{eV}$. Data acquisition is triggered by the LORA scintillator array, which provides energy, arrival direction, and core position estimates of the detected air shower too. Thus only the core of the LOFAR array is currently used for cosmic-ray detection.

In order to extend the energy range of the detected cosmic rays, it is necessary to expand the effective collecting area to the whole LOFAR array. On this purpose, a detailed study about the LOFAR potentialities of working in self-trigger mode, i.e. with the cosmic-ray data acquisition trigger provided by the radio-antenna only, is presented here.

A new method based on the intensity and the frequency spectrum for determining the air shower position to be implemented on LOFAR remote stations is presented too.

35th International Cosmic Ray Conference - ICRC2017

12-20 July, 2017

Bexco, Busan, Korea

${ }^{*}$ Speaker. 


\section{Introduction}

When a cosmic ray interacts with a nucleus of the Earth's atmosphere, an Extensive Air Shower (hereafter "EAS") of secondary particles is started. Associated with the EAS, a radio signal is emitted through Geo-magnetic radiation and Askaryan process. It has been recently proven that the primary particle properties, such as arrival direction, energy, and mass composition, can be reconstructed by ground-based radio detector arrays $[1,2,3]$ with an accuracy similar to current cosmic-ray experiments. Compared to current EAS detectors, which are mainly based on Fluorescence detection or a combined system of particle detectors and fluorescence detectors [5], [6], [7], the main advantage of the Radio-detection technique is its duty cycle up to $100 \%$ and its considerably lower construction and operational costs. On the other hand, given the duration of the radio signal (less than $100 \mathrm{~ns}$ ) and the overwhelming background noise of Radio-Frequency Interferences (hereafter "RFI") for most of the available locations, the direct detection of EAS with radio detectors only has been very difficult so far. An additional issue is set by the need of a very accurate EAS core position reconstruction during the analysis of the radio data. Therefore, particle detectors have often been used in combination with radio antennas.

The LOw-Frequency ARray (LOFAR) [8] is a multipurpose radio antenna array aimed to detect radio signals in the frequency range $10-240 \mathrm{MHz}$. Radio antennas are clustered into over 50 stations, and are spread along Central and Northern Europe, with a higher density in the Northern Netherlands. Each LOFAR station is equipped with 96 omnidirectional Low-frequency Band Antennas (hereafter "LBA"), operational in the $10-90 \mathrm{MHz}$ frequency band, and 96 High-frequency Band Antennas (hereafter "HBA"), operational in the $120-240 \mathrm{MHz}$ frequency band. Both HBA and LBA have two polarization arms (hereafter "dipoles") NE-SW and NO-SE oriented but, due to the different antenna geometry, only LBA can observe the full-sky at once. Therefore, HBA are rarely used for cosmic-ray detection and are not discussed any further in this work. During standard astronomical observations, on each station only half of LBA are active in the so-called "LBA inner" or "LBA outer" configuration, as shown in Fig. 1-left, with a large predominance of LBA outer observations.

The LORA (LOfar Radboud air shower Array) [4] particle detector array covers the region of the LOFAR core, i.e. the region with the highest density of stations, corresponding to a circular area of about $300 \mathrm{~m}$ diameter as shown in Fig. 1-right. The main task of LORA is to provide the trigger to the LOFAR array: when an EAS is detected, the time series data $(\sim 2.2 \mathrm{~ms}$ length, 200 MSample s ${ }^{-1}$ ) of each active antenna polarization arm is downloaded for the offline cosmic-ray analysis. Moreover, the arrival direction, energy, and core position provided by LORA are used as starting values during the following radio data analysis. Since 2011 LOFAR antennas together with LORA have been successfully used for detecting radio-signals associated to EAS in the energy range $10^{16}-10^{18} \mathrm{eV}$ falling within the LOFAR core region [1, 2, 3]. Given the current configuration, only a small fraction of the LOFAR array can be used for cosmic-ray detection. This sets a strong limit to the LOFAR capabilities of detecting cosmic rays at energies above $10^{18} \mathrm{eV}$, where a large coverage area is essential. Unfortunately, expanding the LORA array to cover the full LOFAR array is unfeasible, because of the costs. Moreover, due to the scattered distribution of the LOFAR stations, the radio signal at ground level (hereafter "footprint") of a cosmic ray falling out- 

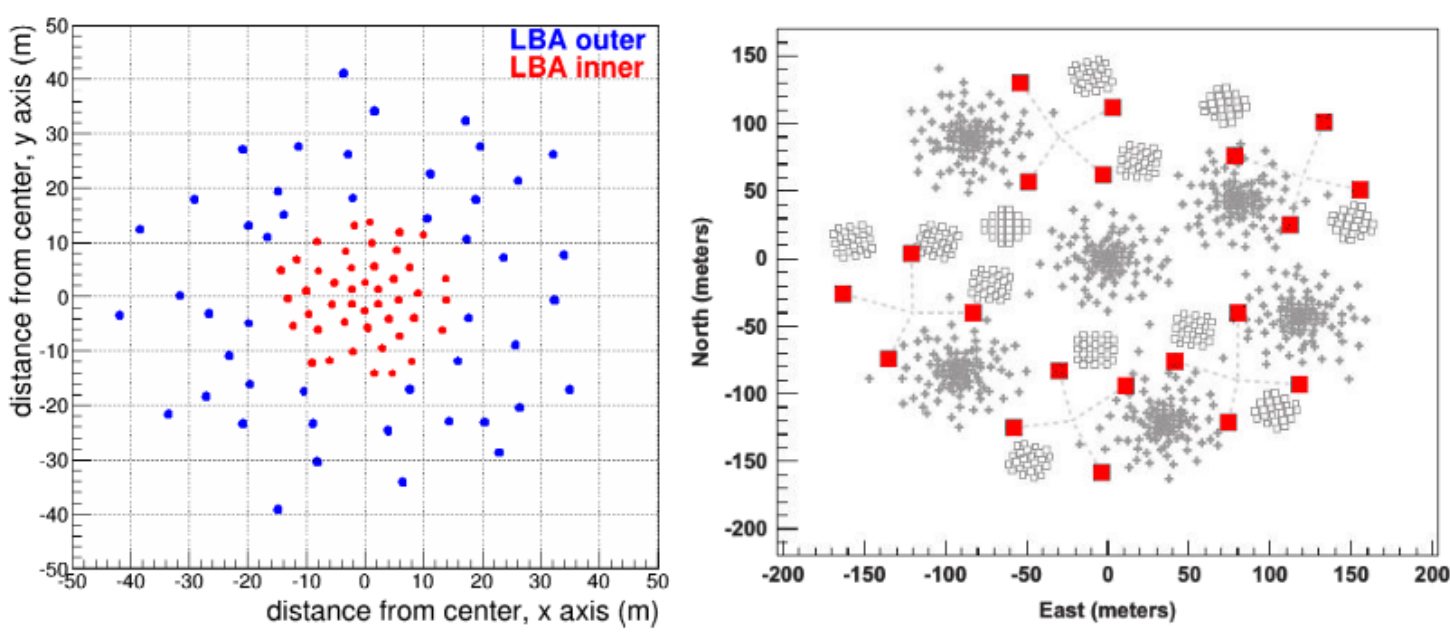

Figure 1: Left: Antenna location in one LOFAR station for LBA inner and LBA outer configuration. Right: LBA (black crosses), HBA (empty squares), and LORA detectors (red squares) position in the LOFAR core region.

side the LOFAR core is quite likely detectable by one station only. Therefore, in order to extend the cosmic-ray detection to the LOFAR remote stations, the following steps have to be implemented:

- a radio self-trigger system: a new trigger system based on the online analysis of the radio data, also known as "radio self-triggering". The solution developed for LOFAR single stations is described in Section 2;

- a single-station EAS core determination technique: the LOFAR cosmic-ray analysis is very sensitive to the position of the EAS core. In case of single-station detection and no particle detector available, the current LOFAR method is not expected to have the required precision. The proposed new EAS core determination technique, based on radio pulse intensity and frequency spectrum, is described in Section 3.

\section{The Radio self-trigger system}

\subsection{The algorithm}

In order to overcome the first addressed issue, an algorithm for triggering individual LOFAR stations has been developed for the online recognition of cosmic rays. The developed algorithm consists of two separate steps: in the first step radio pulses are found ("selection phase"), while in the second one RFI-candidates, i.e. radio pulses which are believed to originate from RFI, are rejected ("RFI rejection phase"). During the selection phase, the radio signal of each dipole is compared with its baseline fluctuation: when a dipole shows a signal above the threshold, that dipole is considered as "fired" . A radio pulse is considered as "detected" if at least 24 antenna dipoles are fired along the same polarization within a coincidence time of $20 \mathrm{~ns}$ in the LBA inner configuration or $30 \mathrm{~ns}$ in the LBA outer configuration. The RFI rejection phase is based on a multi-criteria approach, consisting of evaluating the radio pulse duration, its frequency spectrum, 
its timing, and its arrival zenith angle. Radio pulses satisfying any of the following conditions are treated as RFI and rejected:

- pulse duration: the time window where 3 or more antennas have a signal above threshold within the coincidence time is larger than 75 ns (LBA inner) or 300 ns (LBA outer);

- frequency spectrum: the ratio between the station mean pulse intensity in the $30-45 \mathrm{MHz}$ frequency band and in the $45-70 \mathrm{MHz}$ band is smaller than 1 or larger than 2;

- timing: the pulse occurs within $5 \mu s$ before/after another detected pulse;

- arrival direction: its reconstructed direction of arrival is more than $60^{\circ}$ from the zenith.

If a detected radio pulse passes the RFI rejection phase, the station is considered "triggered" .

\subsection{Analysis and Results}

We considered the raw-data from the 4081 events recorded with LBA outer configuration and the 634 events with LBA inner configuration, corresponding to about $9 \mathrm{~s}$ and $2 \mathrm{~s}$ LOFAR acquisition live-time, respectively. All those events have time, energy, position, and arrival direction successfully reconstructed by LORA, and have at least data for all antennas of one LOFAR station. Stations with one or more missing read-out channels have been discarded.

Four threshold levels, equal to 3, 4, 5, and 10 times the baseline RMS, have been considered for the dipole "firing" condition. The detected radio pulses are considered cosmic-ray signals if occurring within $5 \mu \mathrm{s}$ before and $12.5 \mu \mathrm{s}$ after the LORA time stamp. Any pulse falling outside this time window is considered as RFI pulse. The results obtained for LBA inner and LBA outer configurations are summarized in Table 1 and Table 2.

\begin{tabular}{cccc}
\hline Threshold Level & CR detections & rejected CR detections & RFI triggers after rejection \\
\hline 3 RMS & 395 & $65 \%$ & 0 \\
4 RMS & 246 & $36 \%$ & 0 \\
5 RMS & 168 & $27 \%$ & 0 \\
10 RMS & 51 & $35 \%$ & 0 \\
\hline
\end{tabular}

Table 1: Trigger performances for LBA inner configuration

\begin{tabular}{cccc}
\hline Threshold Level & CR detections & rejected CR detections & RFI triggers after rejection \\
\hline 3 RMS & 1536 & $25 \%$ & 4 \\
4 RMS & 899 & $21 \%$ & 2 \\
5 RMS & 598 & $22 \%$ & 2 \\
10 RMS & 196 & $24 \%$ & 0 \\
\hline
\end{tabular}

Table 2: Trigger performances for LBA outer configuration

For both LBA inner and LBA outer, the primary particle energy distributions of all the analyzed events and of the events generating a trigger in at least one station is shown in Fig. 2. The distributions of the trigger efficiency as function of the distance on the shower plane from the EAS core position is reported in Fig. 3 for the four considered threshold levels together with the detection efficiency of the LOFAR offline analysis. 

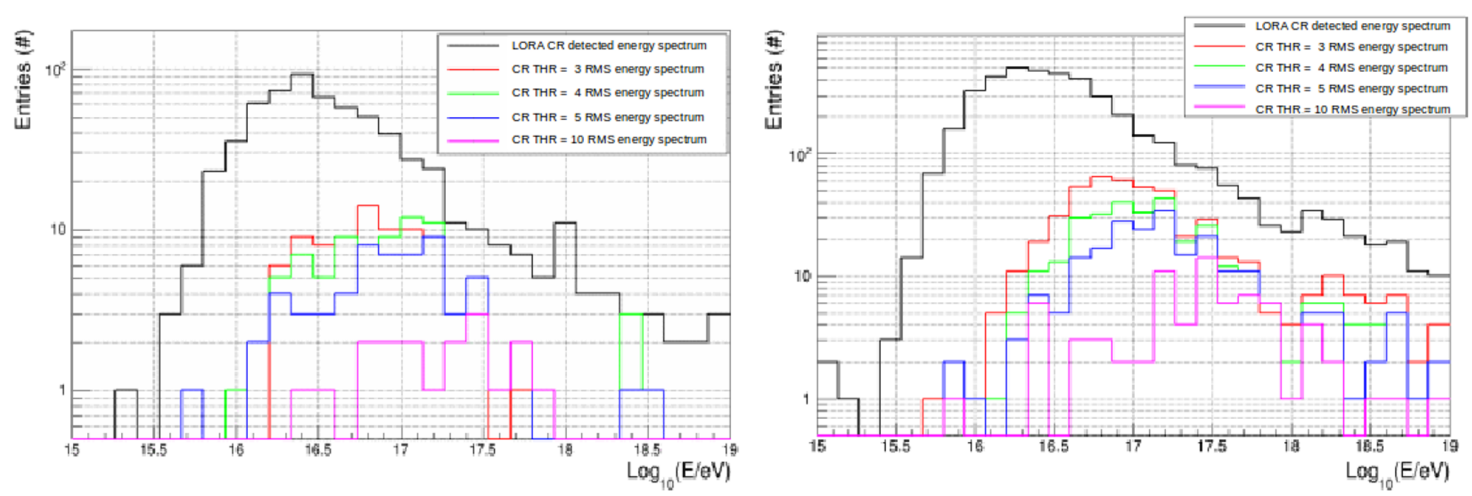

Figure 2: Primary particle energy distributions for LBA inner configuration (left) and LBA outer configuration (right), as reconstructed by LORA. The distribution of the whole set of events is shown together with the distribution of triggered events at trigger threshold equal to 3, 4, 5, and 10 RMS.
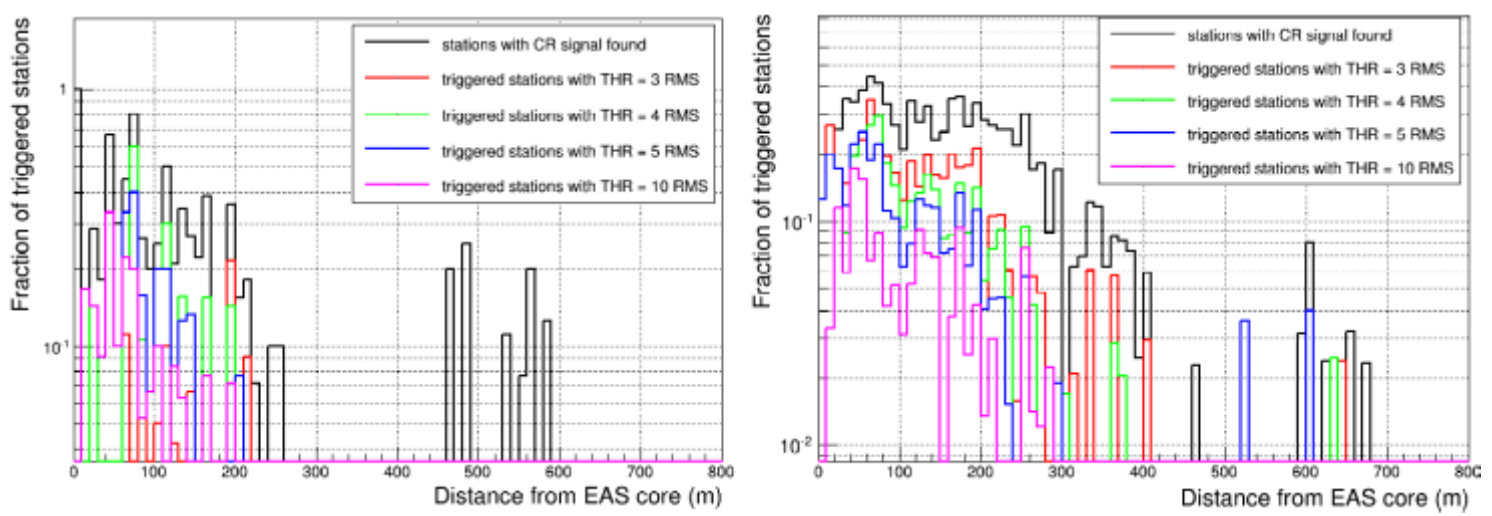

Figure 3: Station trigger efficiency as function of the distance from the LORA reconstructed EAS core position on the shower plane for LBA inner configuration (left) and LBA outer configuration (right) for events of energy above $2 \cdot 10^{17} \mathrm{eV}$. For both distributions the detection efficiency with the LOFAR radio-data offline analysis is reported too.

\section{The EAS core determination}

The current LOFAR method for determining the core position of an EAS triggered by LORA and detected by LOFAR is based on an iterative analysis of the intensity Lateral Distribution Function (hereafter "LDF") of the radio footprint, as discussed in [9] and [2]. The method has been optimized for cosmic rays falling within the LOFAR core region, that means with over 300 available antennas covering an area of approximately $300 \mathrm{~m}$ diameter. That method is not expected to work with the required accuracy for EAS detected by remote stations, where only 48 antennas concentrated on an area of approximately $80 \mathrm{~m}$ diameter are available.

From analytical calculations [10] the frequency spectrum of the radio footprint is expected to be function of the distance from the EAS core. Encouraging results have been obtained on that aspect by the Pierre Auger Observatory [11] and by LOFAR [12]. The new proposed method combines the analysis of the radio-pulse intensity, similarly to what is currently done, with the study of the radio-pulse frequency spectrum. The method is subdivided in the following steps: 
1) pulse timing: on each active antennas, the peak of the sampled signal gives the time of the radio pulse $t_{0}$. Knowing the geometrical position of the antennas and by fitting the distribution of the found $t_{0}$ values with a plane wavefront, the cosmic ray arrival direction is determined;

2) pulse frequency spectrum: the Fast Fourier Transform distribution (hereafter "FFT") is computed for each antenna in a time window of 128 recorded samples, defined as $\left[t_{0}-\right.$ $\left.240 \mathrm{~ns}, t_{0}+400 \mathrm{~ns}\right)$. A linear fit is performed on the logarithm of the FFT squared module distribution, i.e. $\log _{10}|\mathrm{FFT}|^{2}$, in the $33-70 \mathrm{MHz}$ frequency range. The frequency range has been selected to minimize both the RFI-contamination and the border effects of the antenna pass-band filter. The value of the slope $s$ of the linear fit is then extracted;

3) pulse intensity: for each antenna, the intensity of the detected radio pulse is given by the $\mathrm{S} / \mathrm{N}$ parameter, given by the formula

$$
S / N=\frac{\int_{33 \mathrm{MHz}}^{70 \mathrm{MHz}}\left|F F T_{S}\right|^{2}-\left|F F T_{N}\right|^{2} d v}{\int_{33 \mathrm{MHz}}^{70 \mathrm{MHz}}\left|F F T_{N}\right|^{2} d v}
$$

where $\left|\mathrm{FFT}_{\mathrm{S}}\right|^{2}$ is the squared module of the FFT of the radio pulse, as described at previous point, and $\left|\mathrm{FFT}_{\mathrm{N}}\right|^{2}$ is the average of the squared module of the FFT computed in 400 time windows of $640 \mathrm{~ns}$ equally distributed before and after the radio pulse occurrence. Antennas showing $S / N<1$ are discarded from the analysis;

4) parabolic fit: the dependence of the parameter $\zeta(d)$, defined as

$$
\zeta(d)=\frac{\log _{10}(S / N)}{\log _{10}(-s)}
$$

versus the distance from the EAS core on the shower plane $d$ is well represented by a parabolic function By setting the position of the shower core $\left(X_{0}, Y_{0}\right)$ a priori, a parabolic fit of the distribution of the parameter $\zeta(d)$ is performed, and the corresponding $\chi^{2}$ value computed. By repeating the fitting procedure for different $\left(X_{0}, Y_{0}\right)$ positions, the final EAS core position in the shower plane is given by the $\left(X_{0}, Y_{0}\right)$ value minimizing the $\chi^{2}$ value.

In Fig. 4-left the distribution of the $\zeta$ parameter versus the distance from the shower core is shown together its best parabolic fit for one well reconstructed EAS $\left(E=2.9 \cdot 10^{17} \mathrm{eV}\right)$ falling within the LOFAR core region. In Fig. 4-right the $\chi^{2}$ value distribution on the shower plane is reported for the same event, with the obtained EAS core position $\left(\mathrm{X}_{0}, \mathrm{Y}_{0}\right)$ marked with a black star.

\section{Conclusions and Outlook}

A detailed study about the potential to record extensive air showers with LOFAR in selftrigger mode has been carried out. For both LBA inner and LBA outer for cosmic-ray energy above $2 \cdot 10^{17} \mathrm{eV}$, a good trigger efficiency together with a very good RFI-rejection efficiency has been obtained. By considering data acquired during fair-weather conditions only, one RFI pulse 

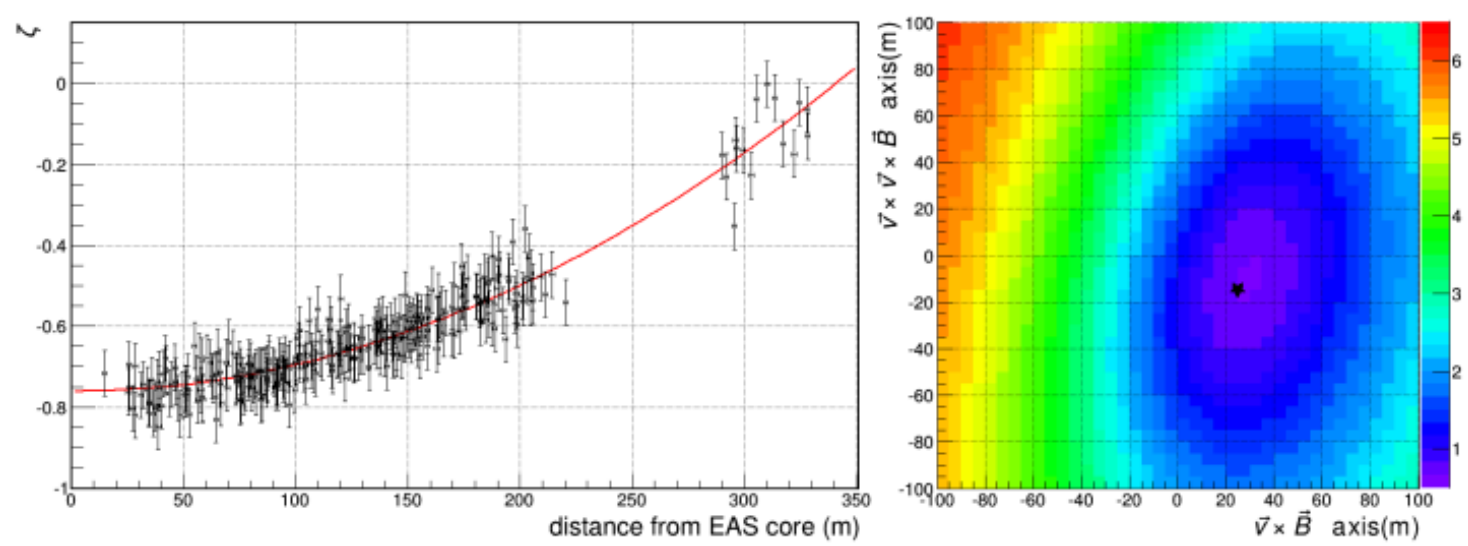

Figure 4: Left: distribution of the $\zeta$ parameter versus the distance from the shower core together its best parabolic fit (in red) for a well reconstructed EAS falling within the LOFAR core region. Right: $\chi^{2}$ distribution of the parabolic fit as function of the EAS core position on the shower plane along the $\vec{v} \times \vec{B}$ and the $\vec{v} \times \vec{v} \times \vec{B}$ directions, where $\vec{v}$ is the cosmic ray moving direction and $\vec{B}$ the Earth's magnetic field. The black star indicates the reconstructed core position. In the shown coordinates system, position $(0,0)$ is the core position as reconstructed by the current LDF analysis.

passed the rejection criteria only (THR $=3$ RMS, LBA outer configuration). However, the available statistics is too low ( $\sim 9$ s LOFAR live-time or $\sim 119$ s summing up all available stations for LBA outer, and $\sim 2 \mathrm{~s}$ LOFAR live-time or $\sim 22 \mathrm{~s}$ summing up all available stations for LBA inner) for concluding that the desired RFI rejection efficiency has been achieved. Low threshold data acquisitions are planned to be performed on one LOFAR test station for increasing the available statistics.

The results obtained about a new air shower reconstruction method are encouraging, even if still preliminary. It has been shown the $\zeta$ parameter can be used for the EAS core position determination. The following step will be to determine the accuracy of the new method compared to the current ones and, afterwards, to apply the new method to single-station cases.

Once the RFI trigger rate is found to be smaller than 1 per hour per station and the new method for the EAS core determination is fine-tuned, the self-trigger algorithm will be implemented to the LOFAR remote stations, in order to extend the LOFAR cosmic-ray collecting area.

\section{Acknowledgments}

The LOFAR cosmic ray key science project acknowledges funding from an Advanced Grant of the European Research Council (FP/2007-2013) / ERC Grant Agreement n. 227610. The project has also received funding from the European Research Council (ERC) under the European Union's Horizon 2020 research and innovation program (grant agreement No 640130). We furthermore acknowledge financial support from FOM, (FOM-project 12PR3041-3) and NWO (Top Grant 614001-454, and Spinoza Prize SPI 78-409). AN is supported by the DFG (research fellowship NE 2031/1-1).

LOFAR, the Low Frequency Array designed and constructed by ASTRON, has facilities in several countries, that are owned by various parties (each with their own funding sources), and that are 
collectively operated by the International LOFAR Telescope foundation under a joint scientific policy.

\section{References}

[1] A. Corstanje et al., The shape of the radio wavefront of extensive air showers as measured with LOFAR, Astroparticle Physics 61 (2015) 22 [astro-ph. HE/1404 . 3907]

[2] A. Nelles et al., The radio emission pattern of air showers as measured with LOFAR - a tool for the reconstruction of energy and shower maximum, Journal of Cosmology and Astroparticle Physics $\mathbf{0 5}$ (2015) 18 [astro-ph.HE/1411.7868]

[3] S. Buitink et al., A large light-mass component of cosmic rays at $10^{17}-10^{17.5}$ electronvolts from radio observations, Nature 531 (2016) 70 [astro-ph. HE/1603.01594]

[4] S. Thoudam et al., LORA: A scintillator array for LOFAR to measure extensive air shower, Nuclear Instruments and Methods in Physics Research Section A 767 (2014) 339 [physics.ins-det/1408.4469]

[5] J. Abraham et al., The fluorescence detector of the Pierre Auger Observatory, Nuclear Instruments and Methods in Physics Research Section A 620 (2010) 227 [astro-ph. IM/ 0907.4282 ]

[6] I. Allekotte et al., The surface detector system of the Pierre Auger Observatory, Nuclear Instruments and Methods in Physics Research Section A 586 (2008) 409 [astro-ph/ 0712 . 2832]

[7] H. Kawai et al., Telescope Array Experiment, Nuclear Physics B (Proceedings Supplements), 175-176 (2008) 221

[8] M. P. Van Haarlem et al., LOFAR: The Low-Frequency ARray, Astronomy \& Astrophysics 556, A2, 53, 2013 [astro-ph. IM/1305.3550]

[9] A. Nelles et al., A parameterization for the radio emission of air showers as predicted by CoREAS simulations and applied to LOFAR measurements, Astroparticle Physics 60 (2015) 13 [astro-ph.HE/1402.2872]

[10] T. Huege and H. Falcke, Radio-Emission from Cosmic Ray Air Showers: Coherent Geosynchrotron Radiation, Astronomy \& Astrophysics 412 (2003) 019 [astro-ph/0 309622 ]

[11] S. Jansen, PhD thesis, Radio for the Masses - Cosmic ray mass composition measurements in the radio frequency domain (2016) ISBN 9789402800739

[12] L. Rossetto et al., Characterisation of the radio frequency spectrum emitted by high energy air showers with LOFAR, in proceedings of $35^{\text {th }}$ ICRC, POS ( ICRC2017) 329 (2017) 